\title{
User Perceptions of the Role and Value of Tags
}

\author{
Yong-Mi Kim \\ School of Information \\ University of Michigan \\ Ann Arbor, MI 48109-1285 \\ kimym@umich.edu
}

\author{
Soo Young Rieh \\ School of Information \\ University of Michigan \\ Ann Arbor, MI 48109-1285 \\ rieh@umich.edu
}

\begin{abstract}
This study investigates user ideas about the role and value of tags in social media. An analysis of 45 interviews with heavy Web users reveals that user perceptions of tags differ from common assumptions held by researchers and designers of social tagging systems. Among beliefs held by participants were that tags were query suggestions or links to other pages, sites, or advertisements - although most identified tags as categories or keywords - and that tags were generated automatically by the computer system. Several participants believed that tags were intended for not only other users but also systems such as search engines. Our findings indicate that Web users, including those who are taggers themselves, experience a high level of uncertainty and confusion about the nature, purpose and value of tags.
\end{abstract}

\section{Author Keywords}

Social tagging, tags, user perceptions, value of tags.

\section{ACM Classification Keywords}

H5.m. Information interfaces and presentation (e.g., $\mathrm{HCI}$ ): Miscellaneous.

\section{General Terms}

Human Factors

\section{INTRODUCTION}

Since its popularization through sites such as Delicious and Flickr, tags have now become part of the landscape of the Web. In recent years many more sites have emerged employing tags as a key feature. These include sites such as YouTube, LibraryThing, and Last.fm, as well as more specialized sites aimed at organizing and sharing academic research, such as Connotea and CiteULike. Tags are also visible on many blogs and newspaper sites.

Currently tags are a feature of the Web to which users are exposed with little or no guidance. For example, a user uploading photographs to Flickr sees an input field for tags, indicating that tags are expected and the user should already

Permission to make digital or hard copies of all or part of this work for personal or classroom use is granted without fee provided that copies are not made or distributed for profit or commercial advantage and that copies bear this notice and the full citation on the first page. To copy otherwise, or republish, to post on servers or to redistribute to lists, requires prior specific permission and/or a fee.

CHI 2011, May 7-12, 2011, Vancouver, BC, Canada.

Copyright 2011 ACM 978-1-4503-0267-8/11/05...\$10.00. know what goes in that text box. In turn, Web pages display tags with no explanation of what they are. However, it is not clear whether Web users, in particular those who have no previous experience attaching tags to online materials, know or understand what tags are.

When discussing tag use, it is helpful to distinguish tag producers and tag consumers, as frequently tag users are understood to be only tag producers, or taggers. In this paper we use the term tag users to refer to both tag consumers and tag producers. Tag producers have been extensively studied on a variety of systems such as Flickr [1,7], Delicious [12], enterprise tag applications [10], and research-motivated systems such as MovieLens [8,9] and MobiTag [2,11]. One of the few studies examining the value of tags to both taggers and non-taggers, [9] found that in general taggers had a more favorable view regarding the usefulness of tags than the overall mix of users. These studies have been valuable in contributing to our understanding of tag producers' perceptions regarding the role and value of tags.

Still, it is unclear whether user perceptions of tags match up with what researchers or designers of social tagging systems had in mind. We examine this issue through the following questions:

1. How do tag users conceptualize tags?

2. To what extent do tag users understand the value of tags?

This study addresses these questions through interviews with Web users, including both tag producers and consumers, without restrictions regarding the specific tagging system(s) they use.

\section{METHODOLOGY}

This paper is based on interview data taken from a larger study that examines people's use of tags during the search process. Semi-structured interviews were conducted with participants following their completion of four search tasks. These tasks involved the use of various search interfaces, which differed as to whether or not tags were present. No explanation or training regarding these interfaces was provided to participants, so as to replicate typical Web use conditions. In the interview after completion of the search tasks, participants were asked questions on their general experience, use, and perception of tags. Participants were first asked to list the Web sites they visit frequently, as well 
as the frequency of their visits. They were then probed about their experience with and their perception of tags on these sites and any additional sites they could recall. At the conclusion of the interview, participants completed a background questionnaire with questions on their demographic information as well as their use of a number of popular tagging sites. Interviews were fully transcribed and analyzed to generate common themes.

\section{Participants}

Forty-five participants who reported having seen tags on the Web were included in this study. Participants were undergraduate students self-identified as native English speakers, recruited through on-campus flyers and Facebook advertisements. They were required to be heavy Web users, but were not required to be tag producers. The intent was to capture the experiences of tag users, without priming them beforehand on tags. These 45 participants (29 women and 16 men) ranged in age from 18 to 23 years old, with a mean age of 20 years. There were 25 different majors represented, from fields including engineering, social and natural sciences, business, and humanities.

In terms of tagging experience, 27 of the 45 participants had tagged on Facebook (15), YouTube (5), blogs (4), Flickr (3), Delicious (2), Twitter (1), and a university library-specific tagging application (1). Of these 27 participants, 13 had only Facebook tagging experience, 12 had non-Facebook tagging experience, and 2 had both. Because of the distinctive nature of tagging on Facebook, which was recognized by participants themselves, we reserve the term "taggers" for just those 14 participants who had experience tagging on systems other than Facebook. Twenty-seven participants were identified as tag consumers, of which 8 participants were pure tag consumers with no tagging experience. Tag consumption included clicking on a tag, as well as examining tags to get additional information or help with query terms. Ten participants could not recall specific instances of producing or consuming tags, but said they had seen and were aware of tags on the web. Our participant pool consisted of a mix of tag producers, tag consumers, and the tag-aware.

\section{RESULTS}

\section{User Conceptualization of Tags}

Three main questions about tags were asked: what are tags, where do they come from, and what is their purpose? The majority of participants supplied site-specific responses regarding tag creators and the purpose of tags, but when defining tags gave answers that applied to all sites.

\section{What are tags according to users?}

Participants were asked to describe tags in their own words. The following ideas of tags emerged from their responses:

- Categories: Tags are a way to group related material together (S30: "I think tags are ways to categorize your

\begin{tabular}{|c|c|c|c|c|}
\hline & Categories & Keywords & Queries & Links \\
\hline Overall & $15(33 \%)$ & $14(31 \%)$ & $7(16 \%)$ & $7(16 \%)$ \\
\hline Taggers & $9(64 \%)$ & $3(21 \%)$ & $1(7 \%)$ & $0(0 \%)$ \\
\hline
\end{tabular}

Table 1. Definition of tags.

information, or information on the Web. Because, on your computer you can make folders and then put your documents into your folder, but on Websites you can't just make a folder and then put it into the folder. So, tags kind of work as folders would work on a regular computer.")

- Keywords: Tags describe key aspects of the document (S39: "crucial words that describe a page")

- Query suggestions: Tags are suggested queries (S41: "related terms that you might wish to check out as well")

- Links: Tags are links to other pages, sites, or advertisements (S29: “They're links that basically take you to articles or sites that have information that is like the name of the tag."; S01: "some of them might be ads.")

The sites mentioned by participants as featuring tags provide some explanation as to why tags were considered to be query suggestions or links to other pages. Several participants mentioned seeing tags on Google, and what they described as tags turned out to be the list of related searches provided at the bottom of the search results. Some participants appeared to consider links in the text of Wikipedia or New York Times articles to be tags. As can be seen in Table 1, query suggestions and links were hardly mentioned by those with tagging experience.

Who or What Creates Tags?

Answers to the question of who or what creates tags were surprisingly diverse. In contrast to their more broadly applicable descriptions of tags, several participants identified different tag creators depending on the tagging system. For example, a participant who said that people uploading videos to YouTube tagged their own videos, also answered that Google generates tags.

Five types of tag sources were identified: content creator, site owner, system, general public, and people paid to tag (Table 2). Content creators or contributors were mentioned in connection with personal blogs and sites such as Flickr or YouTube. Site owners were mentioned for sites such as Engadget and Rotten Tomatoes that do not obviously rely on user-contributed content. Tags were also sometimes perceived as having been automatically generated in some way by "the system," and not by people. The general public, or people tagging content not their own, was mentioned by taggers with experience using Delicious. Several participants said they didn't know or were not sure, 
despite experience tagging on Facebook or using tags to find articles on a specific subject in blogs.

Three of the source types - site owners, system, and people paid to tag - are not typically considered taggers in social tagging research. Forty percent of the participants said they had never tagged anything themselves. Reasons provided included not being aware they could attach tags, and not knowing how to do so for a particular system. This may explain why some respondents did not mention other users like themselves when asked who attaches tags to online content.

\section{What is the purpose of tags?}

Participants were asked what they considered to be the purpose of tags. The purposes that emerged were findability (30 overall, 8 taggers), organization (12 overall, 8 taggers), leading people to content (4 overall, 0 taggers), and description (2 overall, 1 tagger). Several participants identified multiple purposes for tags.

Findability is about making tagged items easier to find with a search engine. Tags are thought of as links between the tagged item and the search engine (S17: "Search engines use tags as a way to reference searches. And people who create Websites use tags to identify to the search engines"). As a consequence, "people can search for it using those specific terms" (S12). In particular, several participants who had uploaded videos to YouTube and tagged these videos said the tags were for the system or search engine, so that their videos would turn up in searches. For these participants, the intended users of tags were search engines, not other people.

Organization is closely related to categorization, or grouping related items together. In this case the intended beneficiaries are people, both tag producers and tag consumers. This type of purpose was generally associated with tags in blogs (S46: "I tagged them because they were recipes ... it was so I could click on something and make all of my recipes come up."). Tags are not for finding the tagged item itself, but for finding things in the same category as the tagged item.

Leading people to content is different from the other two types of purposes described above, in that the intent is not to help people find what they are looking for, but to

\begin{tabular}{|l|c|c|}
\hline & Overall & Taggers \\
\hline Content creator & $26(58 \%)$ & $11(79 \%)$ \\
\hline Site owner & $11(24 \%)$ & $1(7 \%)$ \\
\hline System & $7(16 \%)$ & $2(14 \%)$ \\
\hline General public & $4(9 \%)$ & $3(21 \%)$ \\
\hline People paid to tag & $2(4 \%)$ & $1(7 \%)$ \\
\hline
\end{tabular}

Table 2. Perceived tag creators. manipulate or sometimes mislead people to certain sites by use of tags. These sites can be advertisements or simply misrepresented content. Tags are seen as a way to "string you along" (S5) or "lure people in" (S27), in effect describing tag spam [6].

\section{User understanding of tag value}

Both tag producers and consumers in our study mentioned ignoring tags or not finding them useful. Tags were actively ignored (S14: "I usually just ignore the tags") or not noticed (S32: "I generally don't notice tags."). Some reasons given for ignoring tags were that tags led to content without regard to the user's needs (S36: "it's what they took a picture of and they want me to go look at it. So normally, I just ignore it."), or led to unwanted content (S23: "Tags, in terms of the context bring me to advertisements or lots of pop-ups come out, and I don't really wanna deal with that.") Tags were not considered necessary (S16: "I don't really need tags ... I've never had any use for them."). Contributing to this was the perception that many of the tags were not relevant to the content being tagged (S13: "There are so many tags and it's not that relevant."; S46: "[tags on YouTube] tend to be really bizarre sometimes.").

Tags on blogs were regarded more positively. Participant S46, an avid blog reader, appreciated how tags "take me to large sections of awesome things." She also enjoyed how another blogger used tags for self-expression: "They make me smile because they're just PZ's [blog author's] way of viewing the things that he's responding to." Participants mentioned making use of tags that were topic-specific, as well as those that simply seemed interesting, to explore content in blogs.

Surprisingly, personal experience with tagging sometimes led to a negative perception of the value of tags. For example, for participant S42 exposure to tagging came through a class requiring the use of Delicious. This experience led to a distrust of tags:

S42: "I don't really have a trust for tags yet, because I feel anyone could tag it and it's just your opinion whether it's relevant to a topic or not"

This participant admitted to not expending much effort in tagging, and suspected that other people on the Web were doing likewise. That, in combination with the fact that anybody could tag, meant that tags could not be trusted.

Another participant was led to ignore tags due to "overtagging" by a person close to them:

S27: "I've always been kind of anti-tag and I feel that his [S27's boyfriend] overtagging makes me more so"

For S27, overtagging meant there were a large number of tags due to the inclusion of unnecessary or marginally related tags. She perceived her boyfriend's overtagging in his blog to be about self-presentation, showing how he was interested in diverse topics, and did not consider this to be 
an appropriate use of tags. She herself did not tag her own blog.

\section{DISCUSSION}

In general, our findings reveal that tags are as yet not well understood by Web users, and researchers and system designers should be cautious about assuming that Web users' understanding of tags is similar to their own. While categories and keywords are characterizations commonly found in studies of tagging [3,4,7], our study participants also saw query suggestions and links as tags. We also note that while tags are touted as being "user-generated," they were not uniformly perceived to be as such in our study. Our study participants also mentioned site owners, automatic generation, and people paid to tag in addition to the more typical content creators (e.g., Flickr) or the general public (e.g., Delicious). Participants' beliefs regarding who was doing the tagging varied depending on the site.

A surprising finding was that several participants reported that tags were produced for the consumption of search engines, rather than for other people. This included participants who had experience in uploading and tagging their videos on YouTube. One reason for this perception may be that on YouTube, tags are not visible to users by default. The system requests tags during the video upload, although tags are not visible otherwise, and this may engender the perception that tags are intended for the system, rather than other YouTube users.

Through studies of tag producers, HCI researchers have identified a variety of purposes for tagging, including organization [1,7], finding/search [1,7], self-expression $[2,7]$, reflection or thinking about the tagged items [2], signaling of involvement and expertise [10,11], and adding context or annotation [1]. Our study findings indicate that non-taggers do not see such a rich variety of purposes for tags. The prevalence of YouTube use in our participants (44 out of 45 participants) likely influenced our findings. YouTube, being casual and entertainment-oriented, presents a different use environment than museums or enterprise tagging systems. In addition, YouTube tags have been found to have a number of significant differences from other tagging systems [5].

A limitation of this study is that our sample population of undergraduate students may not be representative of Web users. But, given their age range, participants are so-called "digital natives" for whom the Web has existed their entire lives. They have easy access to computers and Internet connections and are expected to use the Web regularly as part of their school and personal activities. The fact that a population very likely to have encountered tags on the Web had such varying conceptions of tags presents challenges to tagging system designers intending to appeal to wider and more diverse user bases.
The present study indicates the need for a large-scale survey investigating conceptualization and perception of the value of tags. Such a study can reveal perceptions of tagging related to additional social tagging systems not covered in our study. It is important to note that Web users still experience a great deal of uncertainty and confusion about the nature, purpose, and value of tags.

\section{REFERENCES}

1. Ames, M. and Naaman, M. Why we tag: motivations for annotation in mobile and online media. Proc. CHI 2007, ACM Press (2007), 971-980.

2. Cosley, D., Baxter, J., Lee, S., Alson, B., Nomura, S., Adams, P., Sarabu, C., and Gay, G. A tag in the hand: supporting semantic, social, and spatial navigation in museums. Proc. CHI 2009, ACM Press (2009), 19531962.

3. Golder, S.A., and Huberman, B. A. Usage Patterns of Collaborative Tagging Systems. Journal of Information Science, 32, 2 (2006), 198-208.

4. Hammond, T., Hannay, T., Lund, B., \& Scott, J. Social Bookmarking Tools (I): A General Review. D-Lib Magazine, 11, 4 (2005).

5. Heckner, M., Neubauer, T., and Wolff, C. Tree, funny, to_read, google: what are tags supposed to achieve? a comparative analysis of user keywords for different digital resource types. Proc. SSM '08, ACM Press (2008), 3-10.

6. Heymann, P., Koutrika, G., and Garcia-Molina, H. Fighting Spam on Social Web Sites: A Survey of Approaches and Future Challenges. IEEE Internet Computing, 11, 6 (2007), 36-45.

7. Marlow, C., Naaman, M., Boyd, D., and Davis, M. HT06, tagging paper, taxonomy, Flickr, academic article, to read. Proc. Hypertext 2006, ACM Press (2006), 31-40.

8. Sen, S., Lam, S., K. (Tony), Rashid, A. M., Cosley, D., Frankowski, D., Osterhosuse, J. et al. tagging, communities, vocabulary, evolution (N. 4-8, Trans.). Proc. CSCW 2006, ACM Press (2006), 181-190.

9. Sen, S., Harper, F. M., LaPitz, A., \& Riedl, J. The Quest for Quality Tags. Proc. GROUP 2007, ACM Press (2007), 361-370.

10. Thom-Santelli, J., Muller, M.J., and Millen, D.R. Social tagging roles: publishers, evangelists, leaders. Proc. CHI 2008, ACM Press (2008), 1041-1044.

11. Thom-Santelli, J., Cosley, D., and Gay, G. What do you know?: experts, novices and territoriality in collaborative systems, Proc. CHI 2010, ACM Press (2010), 1685-1694.

12. Wash, R. and Rader, E. Public bookmarks and private benefits: An analysis of incentives in social computing. In Proc. ASIS\&T '07, 2007. 